\begin{tabular}{|c|c|}
\hline Title & $\begin{array}{l}\text { Electrochemical Functionalization of InP Porous Nanostructures with a GOD Membrane for A mperometric Glucose } \\
\text { Sensors }\end{array}$ \\
\hline Author(s) & Sato, Taketomo; Mizohata, A kinori; Hashizume, Tamotsu \\
\hline Citation & $\begin{array}{l}\text { Journal of The Electrochemical Society, 157(2), H165-H169 } \\
\text { https://doi.org/10.1149/1.3264634 }\end{array}$ \\
\hline Issue Date & 2010 \\
\hline Doc URL & http:/hdl.handle.net/2115/42543 \\
\hline Rights & $\begin{array}{l}\text { (C) The Electrochemical Society, Inc. 2010. All rights reserved. Except as provided under U.S. copyright law, this work } \\
\text { may not be reproduced, resold, distributed, or modified without the express permission of The Electrochemical Society } \\
\text { (ECS). The archival version of this work was published in J. Electrochem. Soc., V olume 157, Issue 2, pp. H165-H169, } \\
\text { 2010. }\end{array}$ \\
\hline Type & article \\
\hline File Information & JES157_H165.pdf \\
\hline
\end{tabular}

Instructions for use 


\title{
Electrochemical Functionalization of InP Porous Nanostructures with a GOD Membrane for Amperometric Glucose Sensors
}

\author{
Taketomo Sato, ${ }^{*, z}$ Akinori Mizohata, and Tamotsu Hashizume \\ Research Center for Integrated Quantum Electronics and Graduate School of Information Science
} and Technology, Hokkaido University, Sapporo 060-8628, Japan

\begin{abstract}
The electrochemical functionalization of n-type InP porous nanostructures and their feasibility for biochemical sensor applications were investigated. The porous structures have extremely large surface areas, i.e., over $10 \mathrm{~m}^{2} / \mathrm{cm}^{3}$, and superior electrical properties with conductive semiconductor substrates. As a first attempt at electrochemical functionalization, we successfully deposited a glucose oxidase (GOD) membrane onto an InP surface under an applied anodic bias of $1.2 \mathrm{~V}$. With the addition of glucose, the response currents on the porous electrodes increased compared to those on planar InP electrodes due to their enlarged surface area. The sensitivity curves of the porous electrodes we used showed good linearity between the response currents and concentrations in a range from 0 to $5 \mathrm{mM}$.
\end{abstract}

(C) 2009 The Electrochemical Society. [DOI: 10.1149/1.3264634] All rights reserved.

Manuscript submitted July 29, 2009; revised manuscript received September 28, 2009. Published December 7, 2009.

In recent years, the demand for biological and chemical sensors has increased in biochemical field areas such as blood sugar monitoring, antibody and DNA testing, and virus screening. One present challenge in the biochemical sensor research field is to utilize conductive nanomaterials with a large surface area. Various nanomaterials have been investigated for this purpose, such as nanoparticles, ${ }^{1,2}$ carbon nanotubes, ${ }^{3,4}$ semiconductor nanowires, ${ }_{5,6}$ conducting polymers, ${ }^{7}$ and porous materials. ${ }^{8,9}$

Of these, the semiconductor porous nanostructures formed by electrochemical anodization are one of the more promising candidates for use as the building blocks of biochemical sensors. The direct formation of porous structures has been investigated for various materials such as $\mathrm{Si}^{10,11} \mathrm{GaAs},{ }^{12,13} \mathrm{GaP}^{14,15} \mathrm{InP}^{16-19} \mathrm{GaN},{ }^{20}$ and CdSe. ${ }^{13}$ Hamamatsu et al. ${ }^{17}$ succeeded in electrochemically forming arrays of straight nanopores without branches on $\mathrm{n}$-InP(001) substrates. The self-assembled formation of hexagonal arrays of pores were later reported by Fujikura et al. ${ }^{18}$ and Langa et al., ${ }^{19}$ where the pores were laterally separated by InP nanowalls and were formed along a straight vertical direction over a distance of approximately 1-100 $\mu \mathrm{m}$. Such close-packed nanostructures have extremely large surface areas (i.e., over $10 \mathrm{~m}^{2} / \mathrm{cm}^{3}$ ) compared with the semiconductor surfaces prepared by conventional methods used in the semiconductor industry such as lithography and dry etching, vacuum evaporation, and epitaxial growth. Merchant et al. ${ }^{21}$ recently reported that the InP nanowalls between each pore show proper conductivity and carrier mobility. These features reported for InP porous nanostructures are very attractive for biochemical sensor applications.

The purpose of this study is to investigate the feasibility of amperometric biochemical sensors based on $\mathrm{InP}$ porous nanostructures functionalized by enzymatic molecules. In terms of electron mobility, InP is a more suitable semiconductor material for both potentiometric and amperometric sensors than $\mathrm{Si}$, which has been extensively studied using metal-oxide-semiconductor structures. Various gas sensors formed on n-type InP have recently been reported, such as $\mathrm{H}_{2}$ gas sensors ${ }^{22,23}$ and $\mathrm{NO}_{2}$ gas sensors. ${ }^{24}$ They have shown good performance with high sensitivity due to the large change in the conductance responsive adsorption of the target gases. Almost all of the sensors based on InP have utilized bare surfaces or Schottky interfaces to detect the sensing target. To create greater variation in sensing targets, the functionalization process of utilizing organic molecules is important. However, very few efforts have been made to functionalize InP surfaces. ${ }^{25}$ In this study, we first examined the electrodeposition of a glucose oxidase (GOD) membrane on InP planer substrates. We then applied the functionalization

\footnotetext{
* Electrochemical Society Active Member.

${ }^{\text {z }}$ E-mail: taketomo@ rciqe.hokudai.ac.jp
}

process to InP porous nanostructures and investigated their electrocatalytic activity for application to amperometric glucose sensors.

\section{Experimental}

Reagents and materials.- GOD (enzyme class 1.1.3.4, from Aspergillus niger, 200 units/mg) and $\beta-\mathrm{D}-(+)$-glucose were obtained from Sigma-Aldrich Inc. and were used as received. We obtained $0.1 \mathrm{M}$ phosphate buffer solutions (PBSs) with $\mathrm{pH} 7.0$ and 7.4 from Nacalai Tesque Inc. and used them as solvents for the electrodeposition of GOD and for electrochemical measurements, respectively. We obtained $60 \% \mathrm{HNO}_{3}$ and $35 \% \mathrm{HCl}$ solutions from Kishida Chemical Inc. and used them as electrolytes for the formation of porous structures, as described in the literature. ${ }^{26}$ All other solvents and reagents used were of analytical grade, and deionized (DI) water was used to prepare the solutions.

Sn-doped InP(001) substrates (n-type, $n=1 \times 10^{18} \mathrm{~cm}^{-3}$ ) were obtained from Wafer Technology Ltd. The substrates were cleaved into square pieces and used as working electrodes (WEs). The geometric surface area of a WE was defined by the photoresist as $0.13 \mathrm{~cm}^{2}$.

Fabrication of GOD-functionalized InP porous electrodes.The procedures for preparing GOD-functionalized InP porous electrodes are shown in Fig. 1. Electrodes were first anodized at $4 \mathrm{~V}$ in an electrolyte comprising $1 \mathrm{M} \mathrm{HCl}(200 \mathrm{~mL})$ with $\mathrm{HNO}_{3}(3 \mathrm{~mL}){ }^{26}$ Here, pore diameter and wall thickness can be controlled by adjusting the electrochemical anodic and cathodic conditions. ${ }^{27}$ After that the porous surface was photoelectrochemically etched in the same electrolyte under illumination to remove the disordered irregular layer formed at the initial stage of pore formation. ${ }^{28}$ Figure $2 \mathrm{a}$ and $\mathrm{b}$ shows typical scanning electron microscope (SEM) images of an InP porous sample after photoelectrochemical (PEC) etching for 200 s. Regular-sized pores of about $130 \mathrm{~nm}$ in diameter appeared on the surface over a wide area. The pore depth $d$ was $3.2 \mu \mathrm{m}$ from the SEM observations of the cross section. Finally, we conducted an electrodeposition of GOD in a $10 \mathrm{mg} / \mathrm{mL}$ GOD-containing electrolyte based on a PBS with $\mathrm{pH}$ 7.0. After electrodeposition, each sample was washed with DI water and stored under dry conditions in a refrigerator.

Characterization and amperometric measurements.- A series of electrochemical functionalization and amperometric measurements was conducted using a standard cell with three electrodes: an InP porous electrode used as a WE, a Pt counter electrode, and a saturated calomel electrode (SCE) used as a reference. The WE potential with respect to SCE was precisely controlled with a potentiostat (Princeton Applied Research 273 A).

First, we attempted to optimize the conditions for the electrodeposition of GOD onto the InP surfaces. For this purpose, an $\mathrm{InP}(001)$ planar electrode was used as a WE that was dipped into a 


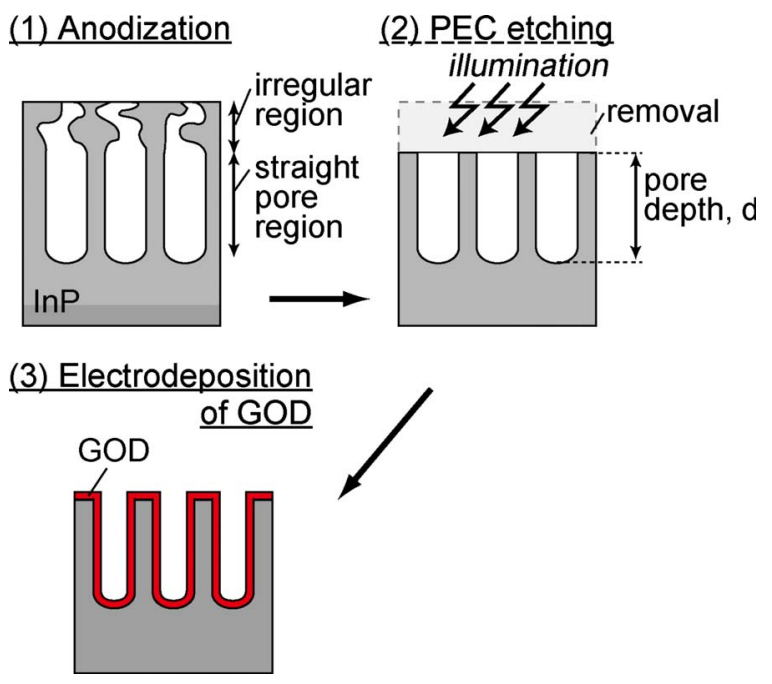

Figure 1. (Color online) Scheme for the preparation of a GODfunctionalized InP porous biosensor. Step 1: electrochemical formation of InP porous nanostructures. Step 2: removal of the irregular top layer by PEC etching. Step 3: electrodeposition of GOD.

GOD-containing electrolyte. The applied bias for the electrodeposition $V_{\mathrm{d}}$ was varied over a range from 0 to $1.2 \mathrm{~V}$, and the deposition time was set at $60 \mathrm{~s}$. The GOD membrane formed on the InP surface was analyzed by X-ray photoelectron spectroscopy (XPS) using a Perkin-Elmer PHI 1600 with a monochromatic Al K $\alpha$ source at $1486 \mathrm{eV}$.

We then investigated the sensing properties of the GODfunctionalized InP porous electrodes used as an amperometric glucose sensor. The electrodes were dipped into a PBS with $\mathrm{pH}$ 7.4, and a sensing bias $V_{\mathrm{s}}$ was set at $+0.65 \mathrm{~V}$ under darkroom conditions. Mild magnetic stirring was maintained throughout the measurements. After the background current stabilized, amperometric measurements were carried out by successively adding a glucose solution into the PBS and recording the current signals.

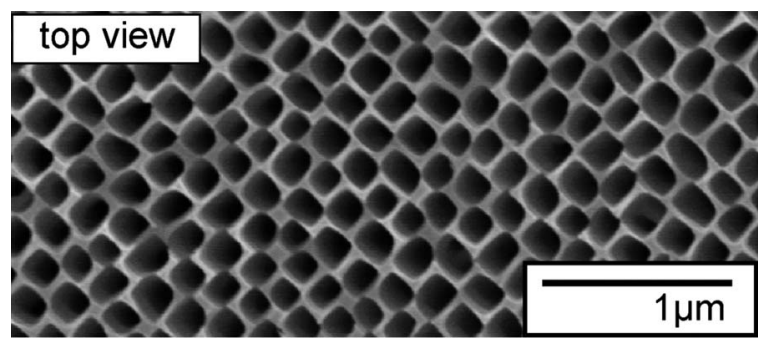

(a)

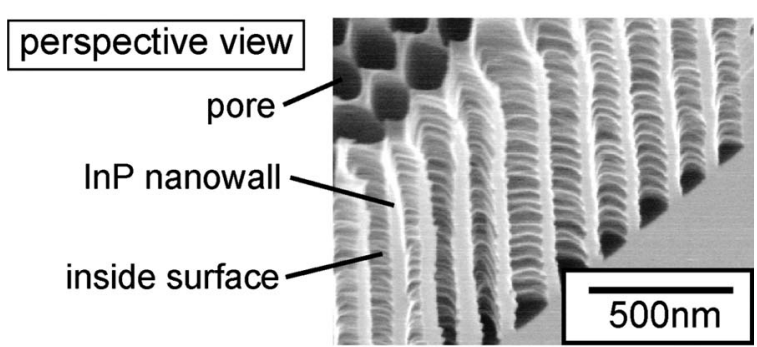

(b)

Figure 2. SEM images of InP porous structures after a PEC process. (a) Top view of the (001) surface and (b) perspective view with cross section.

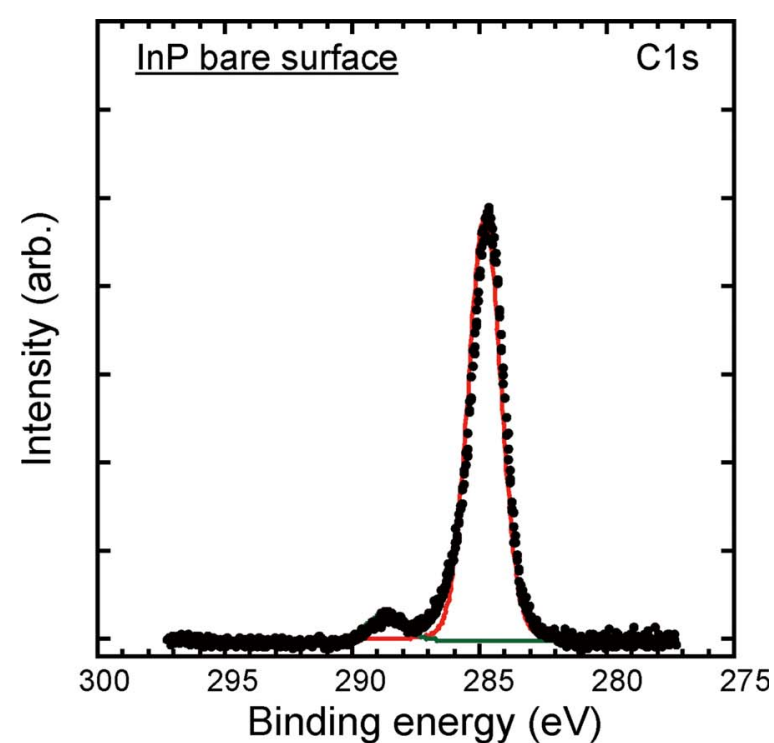

(a)

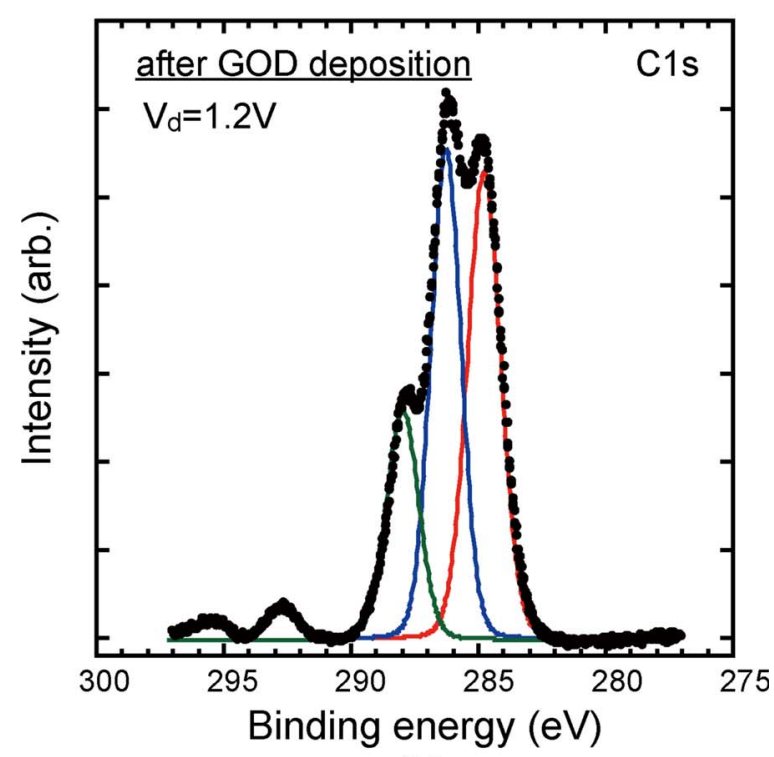

(b)

Figure 3. (Color online) C 1s XPS spectra obtained from (a) the InP bare surface and (b) the GOD-deposited InP surface at $V_{\mathrm{d}}$ of $1.2 \mathrm{~V}$.

\section{Results and Discussion}

Electrodeposition of the GOD membrane onto an InP surface.Figure $3 \mathrm{a}$ and $\mathrm{b}$ shows the $\mathrm{C} 1 \mathrm{~s}$ XPS spectra obtained from the reference InP bare surface and the GOD-deposited surface at $V_{\mathrm{d}}$ of $1.2 \mathrm{~V}$. As shown in Fig. 3a, a sharp peak was observed at $285 \mathrm{eV}$ in the hydrocarbon $(\mathrm{C}-\mathrm{H})$ region, and a small and broad oxide peak was observed at a higher energy. A more complicated spectrum was observed from the GOD-deposited InP surface, as shown in Fig. 3b. Simple apportionment of the $\mathrm{C} 1 \mathrm{~s}$ spectrum was enabled by the amidic characteristics of GOD with two specific peaks at 288 and $286 \mathrm{eV}$, corresponding to those of carbonyl and $\mathrm{N}-\mathrm{C}-\mathrm{CO}$ groups, respectively. ${ }^{29}$ These groups were clearly absent in the $\mathrm{C} 1 \mathrm{~s}$ spectrum obtained from the InP bare surface. These results confirmed that a GOD membrane was formed on the InP surface by the electrodeposition process.

To optimize the electrochemical conditions, XPS analysis was performed on the deposited GOD membranes by changing the anodic bias applied to the InP substrates. Figure 4 compares the ratios 


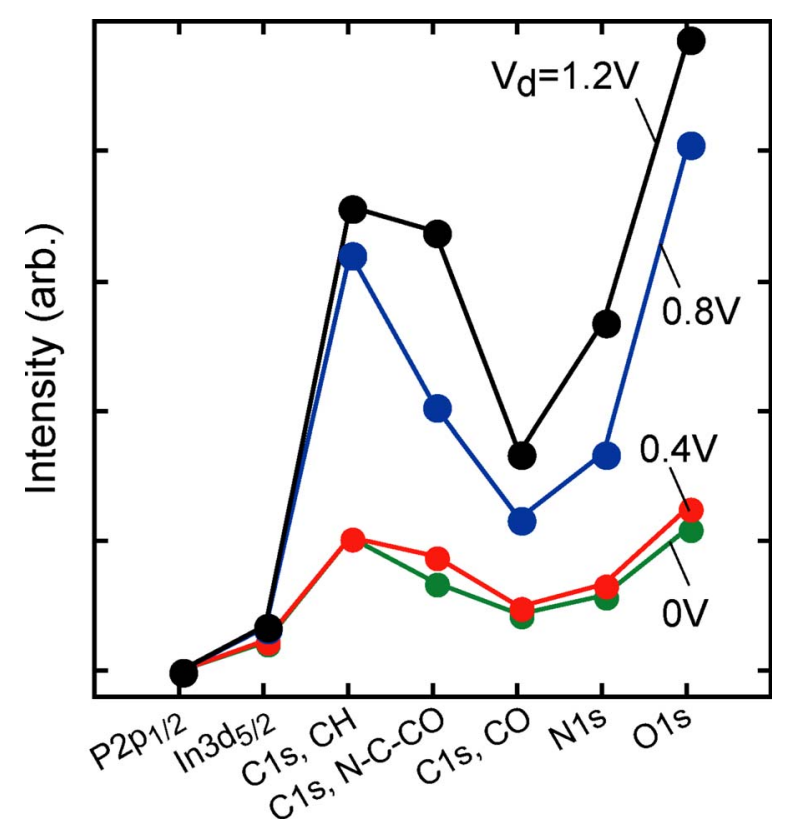

Figure 4. (Color online) XPS peak area ratio of $\mathrm{C} 1 \mathrm{~s}, \mathrm{~N} 1 \mathrm{~s}$, and $\mathrm{O} 1 \mathrm{~s}$ to $\mathrm{P}$ $2 \mathrm{p}_{1 / 2}$ on GOD-deposited surfaces as a function of the anodic bias applied.

of the XPS peak areas of various elements $(\mathrm{C} 1 \mathrm{~s}, \mathrm{~N} 1 \mathrm{~s}$, and $\mathrm{O} 1 \mathrm{~s})$ to $\mathrm{P} 2 \mathrm{p}_{1 / 2}$ that originated in the InP substrates. For the sample prepared with an anodic bias of $0.4 \mathrm{~V}$, the XPS ratios showed no significant changes from those obtained with the reference sample. However, as the anodic bias was increased to $0.8 \mathrm{~V}$, the ratios of GOD-related elements such as $\mathrm{C}, \mathrm{N}$, and $\mathrm{O}$ increased. As shown in Fig. 4, the sample prepared with an anodic bias of $1.2 \mathrm{~V}$ had a more pronounced tendency to show increased ratios of GOD-related elements. These results suggest that a GOD membrane was efficiently deposited on the InP surface at a higher anodic bias. The biasdependent XPS spectra can be explained as follows. In this study, the electrolyte $\mathrm{pH}$ was adjusted to 7.0, which is higher than the isoelectric point for GOD $(p \mathrm{I}=4.2)$. Because the GOD molecules were negatively charged in the electrolyte, the electric force that draws the GOD toward the InP surface was increased under the anodic bias applied.

InP surfaces are readily oxidized in an aqueous electrolyte under an anodic bias applied even under darkroom conditions. ${ }^{30}$ A higher anodic bias increases the risk of inactivating the GOD molecules due to the protein denaturation caused by the high electric field at the electrolyte/InP surface. For the same reason, Matsumoto et al. ${ }^{31}$ reported that the anodic bias of $1.3 \mathrm{~V}$ (vs $\mathrm{AgCl} / \mathrm{Ag}$ electrode) is suitable for the GOD deposition onto the Pt surface. Thus, we decided to use the anodic bias of $1.2 \mathrm{~V}$ for our next experiments on the electrodeposition of GOD onto an InP porous surface.

Glucose detection by InP porous electrode.- The electrocatalytic activity of InP porous electrodes was investigated after the electrodeposition of the GOD membrane at $V_{\mathrm{d}}$ of $1.2 \mathrm{~V}$. Figure $5 \mathrm{a}$ compares the current response of the porous electrodes with a pore depth $d$ of 3.2 or $1.9 \mu \mathrm{m}$ and that of a reference planar electrode $(d=0 \mu \mathrm{m})$ to which glucose solution was added to the PBS with $\mathrm{pH}$ 7.0. The surface area used to calculate the current density comprises only the geometric surface area and does not include the wall inside. For planar electrodes with a GOD membrane, almost no response current was observed. However, anodic response currents were clearly observed on InP porous electrodes, as shown in Fig. 5a. As the amount of glucose solution increased as indicated by the arrows, the anodic current increased. The current level obtained from the sample with a $d$ of $3.2 \mu \mathrm{m}$ was larger than that obtained

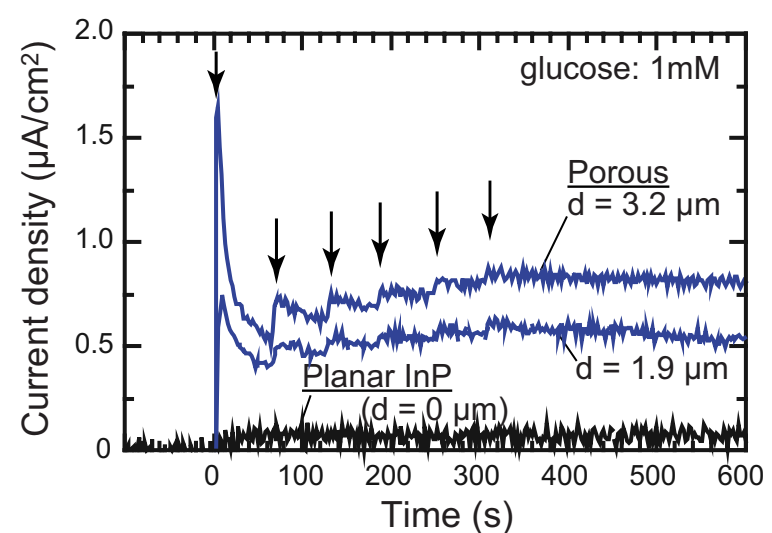

(a)

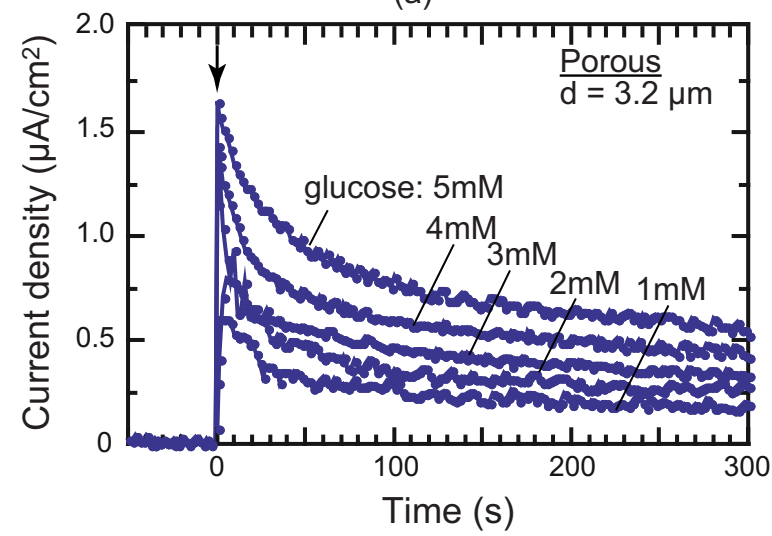

(b)

Figure 5. (Color online) (a) Current response of the porous and reference planar electrodes to the addition of glucose. Arrows indicate the timing of the addition of glucose into the PBS electrolyte. (b) Transient curves of anodic currents obtained on the porous electrode with different concentrations of glucose only added once.

from the sample with a $d$ of $1.9 \mu \mathrm{m}$. This suggests that the electrocatalytic activity of the InP porous electrodes was enhanced due to the increased surface area with pore depth $d$.

Furthermore, the response current reached peak values at the very beginning of the glucose addition process and decreased thereafter. Figure $5 \mathrm{~b}$ shows the transient curves of anodic currents obtained on a porous electrode with a $d$ of $3.2 \mu \mathrm{m}$ by adding different concentrations of glucose at a time. As expected, the measured current density was strongly dependent on the glucose concentration. After the currents reached peak values at the very beginning, they then decreased rapidly and approached various saturation values, as shown in Fig. 5b. This kind of transient current behavior has not been previously observed in planar electrodes on a time scale of seconds to minutes. The current response we observed is most probably due to the diffusion-limited process of reactants inside nanopores with a small diameter.

Glucose in electrolytes can be detected by a GOD-functionalized electrode with the following reactions

$$
\begin{gathered}
\text { glucose }+\mathrm{O}_{2} \rightarrow \text { glucono - lactone }+\mathrm{H}_{2} \mathrm{O}_{2} \\
\mathrm{H}_{2} \mathrm{O}_{2} \rightarrow \mathrm{O}_{2}+2 \mathrm{H}^{+}+2 \mathrm{e}^{-}
\end{gathered}
$$

GOD catalyzes the conversion of a glucose molecule, as described in Eq. 1, where one glucose molecule reacts with one waterdissolved oxygen molecule generating one molecule of hydrogen peroxide $\left(\mathrm{H}_{2} \mathrm{O}_{2}\right)$. When an anodic bias was applied to a sensor electrode large enough to induce the reaction described in Eq. 2, anodic currents were observed as response currents. We have recently reported that $\mathrm{H}_{2} \mathrm{O}_{2}$ can be detected by an InP porous elec- 


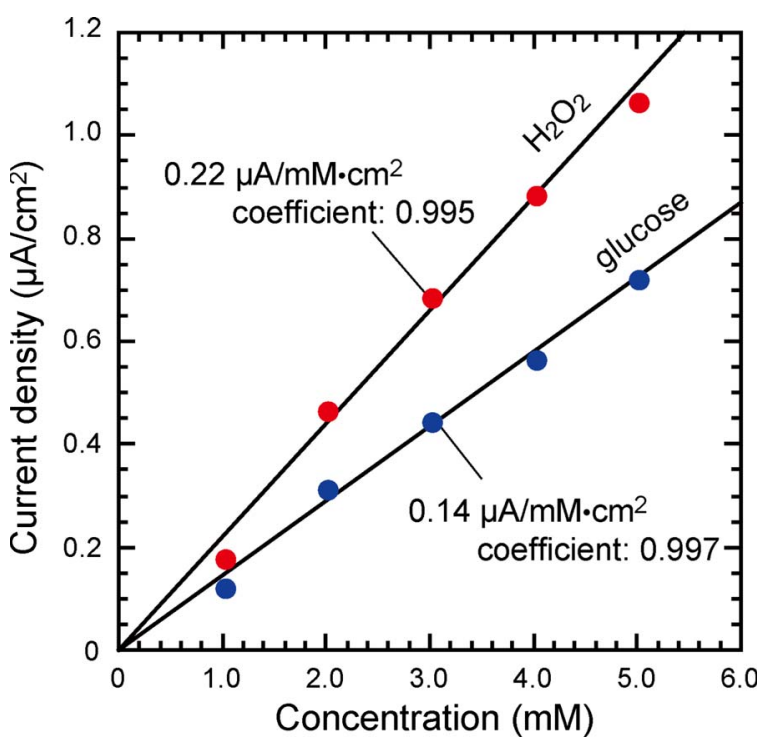

Figure 6. (Color online) Calibration plots of anodic currents vs electrolyte concentration for the detection of $\mathrm{H}_{2} \mathrm{O}_{2}$ and glucose.

trode under applied $V_{\mathrm{s}}$ of $0.65 \mathrm{~V}$ (i.e., the same bias we applied for the measurements taken in this work). ${ }^{32}$ In this study, the GODfunctionalized porous electrode detected the glucose added to the electrolyte in the form of increased currents for the anodization of $\mathrm{H}_{2} \mathrm{O}_{2}$ produced by the reaction described in Eq. 1 .

The current transient properties observed here may involve the diffusion process of reactants such as $\mathrm{H}_{2} \mathrm{O}_{2}$ and $\mathrm{O}_{2}$ inside the nanopores with a high aspect ratio. As shown in Fig. 5a, subsequent response currents considerably decreased after the first addition of $\mathrm{H}_{2} \mathrm{O}_{2}$. This suggests that the subsequent supply of $\mathrm{H}_{2} \mathrm{O}_{2}$ was inhibited by the residual reactant produced at the first reaction in the nanopores. However, further experimental and theoretical discussions are necessary to clarify the complicated current response observed using the porous electrodes.

Sensitivity and stability of InP porous sensors.-As shown in Fig. 5a, we found that the sensitivity for the present InP porous electrodes strongly depends on the pore depth $d$ because the effective surface area inside the pores increases with $d$. This suggests that the InP nanowalls between pores are conductive and that the wall surface effectively behaves as an electrode surface. However, one might think that the surface depletion would prevent the current flow due to the decreased thickness of the conductive nanowalls. In the present porous structures, the thickness of the InP nanowalls was 55 $\mathrm{nm}$ on average and the depletion layer thickness was estimated at about $25 \mathrm{~nm}$ if the surface conditions of a bulk InP are directly applicable to the nanowall. Merchant et al. investigated the conductivity of nanoporous InP membranes with comparable size to ours and obtained proper conductivity in the InP nanowalls. ${ }^{21}$ This report supports the results we have obtained on the sensor ability of InP porous structures.

The calibration plots for detecting glucose and $\mathrm{H}_{2} \mathrm{O}_{2}$ obtained with $\mathrm{InP}$ porous electrodes are compared in Fig. 6. The values for the current density were measured at $t=60 \mathrm{~s}$ after glucose or $\mathrm{H}_{2} \mathrm{O}_{2}$ was added. All data plotted in Fig. 6 were calibrated by subtracting zero-offset currents from the experimental data obtained from the measurements. Both plots exhibit good linearity between the current signal and concentrations in a range from 0 to $5.0 \mathrm{mM}$. The correlation coefficients obtained by linear fitting on the experimental data were greater than or equal to 0.995 . As shown in Fig. 6, the sensitivity of $\mathrm{H}_{2} \mathrm{O}_{2}$ detection was $0.22 \mu \mathrm{A} / \mathrm{mM} \mathrm{cm}{ }^{2}$. A lower sensitivity of $0.14 \mu \mathrm{A} / \mathrm{mM} \mathrm{cm}{ }^{2}$ was obtained for the same electrode when testing it for glucose detection. One possible explanation for this difference is that the response speed was limited by the enzyme reaction described in Eq. 2, whose reaction rate was much lower than that described in Eq. 1.

The sensitivity for detecting glucose obtained in this study was higher than $0.023 \mu \mathrm{A} / \mathrm{mM} \mathrm{cm}{ }^{2}$ that was previously obtained for carbon-paste electrodes. ${ }^{33}$ Carbon-related materials have been investigated earlier as a sensor material due to their high potential abilities in terms of both surface area and electron mobility. In consideration of this point, our data obtained for the InP porous nanostructures was not bad for an initial attempt. However, our sensor structure has not been optimized as compared with the recent progress on carbon nanotubes. Xie et al. reported that a higher sensitivity of $52.7 \mu \mathrm{A} / \mathrm{mM} \mathrm{cm}^{2}$ was obtained for Pt-coated multiwalled carbon nanotube electrodes. ${ }^{3}$ Improved sensitivity of the InP porous electrodes can be possibly obtained by optimizing such structural properties as pore depth and pore diameter and by using $\mathrm{Pt}$ nanoparticles to enhance functionalization inside the pores.

The data shown in Fig. 6 were obtained by using the same electrode repeatedly while changing its electrolyte. The results indicate that the present InP porous electrodes are stable enough to obtain reproducibly increased currents in response to the glucose concentration. However, the performance of the electrodes degraded for a few weeks during which the response currents decreased by about $30 \%$ compared to the data obtained at the first measurement. This is probably due to the deactivation of the GOD membrane, where we had not considered surface protection as a first step for the functionalization of the InP porous nanostructures with a GOD membrane. We suppose that InP itself is chemically stable in terms of biochemical applications. We have recently reported that InP-based ionsensitive field effect transistors showed a stable operation of a liquid-phase $\mathrm{pH}$ sensor in diluted $\mathrm{HCl}, \mathrm{H}_{3} \mathrm{PO}_{4}$, and $\mathrm{NaOH}$ electrolytes in a $\mathrm{pH}$ range from 3.0 to $12.0 .{ }^{34}$ This covers the optimum $\mathrm{pH}$ range for various enzymatic reactions, such as lipase ( $\mathrm{pH} 4.0-5.0)$, amylase (pH 6.7-7.0), and trypsin ( $\mathrm{pH} 7.8-8.7) .{ }^{35}$ From our present results described here, we consider that InP porous electrodes functionalized by electrodeposition have considerable potential for practical application to various amperometric biochemical sensors.

\section{Conclusion}

We investigated the functionalization process of n-type InP porous electrodes and their feasibility for biochemical sensor applications. As a first attempt at achieving electrochemical functionalization, a GOD membrane was successfully deposited onto an InP surface under an applied anodic bias. The response currents of the glucose sensors increased on the porous InP electrodes due to their enlarged surface area. The functionalized porous electrodes exhibited good sensitivity with respect to detecting glucose concentration in the electrolyte, where the response currents detected were attributed to $\mathrm{H}_{2} \mathrm{O}_{2}$ anodization. We consider that the present porous electrodes functionalized by electrodeposition have considerable potential for practical application to various amperometric biochemical sensors.

\section{Acknowledgments}

The work reported here was supported in part by a Grant-in-Aid for Young Scientists (A)-21686028 from the Japanese Ministry of Education, Culture, Sports, Science, and Technology. article.

Hokkaido University assisted in meeting the publication costs of this

\section{References}

1. Z. W. Zhao, X. J. Chen, B. K. Tay, J. S. Chen, Z. J. Han, and K. A. Khor, Biosens Bioelectron., 23, 135 (2007).

2. S. Tokonami, H. Shiigi, and T. Nagaoka, J. Electrochem. Soc., 155, J105 (2008)

3. J. Xie, S. Wang, L. Aryasomayajula, and V. K. Varadan, Nanotechnology, 18, 062203 (2007)

4. M. D. Shirsat, C. O. Too, and G. G. Wallace, Electroanalysis, 20, 150 (2008)

5. Y. Cui, Q. Wei, H. Park, and C. M. Lieber, Science, 293, 1289 (2001).

6. A. Ganguly, C.-P. Chen, Y.-T. Lai, C.-C. Kuo, C.-W. Hsu, K.-H. Chen, and L.-C. Chen, J. Mater. Chem., 19, 928 (2009).

7. M. A. Bangar, D. J. Shirale, W. Chen, N. V. Myung, and A. Mulchandani, Anal. 
Chem., 81, 2168 (2009)

8. M. S. Salem, M. J. Sailor, F. A. Harraz, T. Sakka, and Y. H. Ogata, J. Appl. Phys., 100, 083520 (2006).

9. H. Qiu, L. Xue, G. Ji, G. Zhou, X. Huang, Y. Qu, and P. Gao, Biosens. Bioelectron., 24, 3014 (2009).

10. R. L. Smith, S.-F. Chuang, and S. D. Collins, J. Electron. Mater, 17, 533 (1988).

11. L. T. Canham, Appl. Phys. Lett., 57, 1046 (1990).

12. P. Schmuki, J. Fraser, C. M. Vitus, M. J. Graham, and H. S. Isaacs, J. Electrochem. Soc., 143, 3316 (1996)

13. I. M. Tiginyanu, V. V. Ursaki, E. Monaico, E. Foca, and H. Föll, Electrochem. Solid-State Lett., 10, D127 (2007).

14. A. Anedda, A. Serpi, V. A. Karavanskii, I. M. Tiginyanu, and V. M. Ichizli, Appl. Phys. Lett., 67, 3316 (1995).

15. K. Kuriyama, K. Ushiyama, K. Ohbora, Y. Miyamoto, and S. Takeda, Phys. Rev. B, 58, 1103 (1998)

16. T. Takizawa, S. Arai, and M. Nakahara, Jpn. J. Appl. Phys., Part 2, 33, L643 (1994).

17. A. Hamamatsu, C. Kaneshiro, H. Fujikura, and H. Hasegawa, J. Electroanal. Chem., 473, 223 (1999).

18. H. Fujikura, A. Liu, A. Hamamatsu, T. Sato, and H. Hasegawa, Jpn. J. Appl. Phys., Part 1, 39, 4616 (2000).

19. S. Langa, I. M. Tiginyanu, J. Carstensen, M. Christophersen, and H. Föll, Appl. Phys. Lett., 82, 278 (2003).

20. M. Mynbaeva, A. Titkov, A. Kryganovskii, V. Ratnikov, K. Mynbaev, H. Hu- htinen, R. Laiho, and V. Dmitriev, Appl. Phys. Lett., 76, 1113 (2000).

1. S. K. E. Merchant, J. L. Hughes, L. Sirbu, I. M. Tiginyanu, P. Parkinson, L. M. Herz, and M. B. Johnston, Nanotechnology, 19, 395704 (2008).

22. H.-I. Chen, Y.-I. Chou, and C.-Y. Chu, Sens. Actuators B, 85, 10 (2002).

23. T. Kimura, H. Hasegawa, T. Sato, and T. Hashizume, Jpn. J. Appl. Phys., Part 1, 45, 3414 (2006).

24. K. Wierzbowska, L. Bideux, B. Adamowicz, and A. Pauly, Sens. Actuators, A, 142, 237 (2008)

25. H. H. Park and A. Ivanisevic, J. Phys. Chem. C, 111, 3710 (2007).

26. T. Sato, T. Fujino, and H. Hasegawa, Appl. Surf. Sci., 252, 5457 (2006).

27. T. Sato, T. Fujino, and T. Hashizume, Electrochem. Solid-State Lett., 10, H153 (2007).

28. T. Sato and A. Mizohata, Electrochem. Solid-State Lett., 11, H111 (2008).

29. A. Griffith, A. Glidle, and J. M. Cooper, Biosens. Bioelectron., 11, 625 (1996).

30. C. Kaneshiro, T. Sato, and H. Hasegawa, Jpn. J. Appl. Phys., Part 1, 38, 1147 (1999).

31. N. Matsumoto, X. Chen, and G. S. Wilson, Anal. Chem., 74, 362 (2002).

32. T. Sato, A. Mizohata, N. Yoshizawa, and T. Hashizume, Appl. Phys. Express, 1, 051202 (2008).

33. J. Wang, L. H. Wu, Z. Lu, R. Li, and J. Sanchez, Anal. Chim. Acta, 228, 251 (1990).

34. N. Yoshizawa, T. Sato, and T. Hashizume, Jpn. J. Appl. Phys., 48, 091102 (2009).

35. B. Harrow and A. Mazur, Textbook of Biochemistry, p. 104, Saunders, Philadelphia, PA (1966). 